\title{
EXTENDED HESITANT FUZZY SETS
}

\author{
Bin ZHU ${ }^{\mathrm{a}}$, Zeshui XU \\ ${ }^{a}$ School of Economics and Management, Tsinghua University, Beijing 100084, China \\ ${ }^{b}$ Business School, Sichuan University, Chengdu, Sichuan 610064, China
}

Received 02 September 2012; accepted 22 June 2013

\begin{abstract}
Hesitant fuzzy sets (HFSs) are a useful tool to manage situations in which the decision makers (DMs) hesitate about several possible values for the membership to assess a variable, alternative, etc. However, HFSs have the information loss problem and cannot identify different DMs, which interferes with the application of HFSs in decision making. To overcome these limitations, we develop the extended hesitant fuzzy sets (EHFSs) in this paper. As an extension of HFSs, EHFSs have close relationships with existing fuzzy sets including intuitionistic fuzzy sets (IFSs), fuzzy multisets (FMSs), type-2 fuzzy sets (T2FSs), dual hesitant fuzzy sets (DHFSs), and especially HFSs. We propose a concept of extended hesitant fuzzy elements (EHFEs), then study the basic operations and the desirable properties of EHFEs in detail. Some extended hesitant distance measures are developed to illustrate their advantages comparing with the existing hesitant distance measures. To extend EHFSs to decision making, we combine the proposed distance measures with the Dempster-Shafer belief structure.
\end{abstract}

Keywords: extended hesitant fuzzy sets (EHFSs), extended hesitant fuzzy elements (EHFEs), hesitant fuzzy sets (HFSs), hesitant fuzzy elements (HFEs), distance measure, decision making.

JEL Classification: D81, D7.

\section{Introduction}

Zadeh (1965) introduced fuzzy sets (FSs) as a powerful tool to address fuzziness, which have wide applications in practice (Baležentis et al. 2012; Stankevičienė, Mencaite 2012). Then researchers developed some extensions of FSs, such as intuitionistic fuzzy sets (IFSs) (Atanassov 1986), type-2 fuzzy sets (T2FSs) (Zadeh 1975; Mizumoto, Tanaka 1976; Dubois 1980), fuzzy multisets (FMSs) (Yager 1986), interval-valued fuzzy sets (IVFSs) (Zadeh 1975), interval-valued intuitionistic fuzzy sets (IVIFSs) (Atanassov, Gargov 1989), hesitant fuzzy sets (HFSs) (Torra 2010) and dual hesitant fuzzy sets (DHFSs) (Zhu et al. 2012b).

Corresponding author Bin Zhu, Zeshui Xu

E-mail: binzhu@263.net; xuzeshui@263.net 
Among these sets, IFSs should be one of the most famous extensions of FSs due to the simultaneous consideration of the membership and non-membership. From a mathematical point of view, IFSs, modeled with two functions that define an interval, can also be considered as IVFSs. Actually, IFSs and IVFSs are equipollent generalizations of the FSs. Atanassov and Gargov (1989) then proposed IVIFSs as another generalization of IFSs (Xu et al. 2008; Wang et al. 2009; Xu 2010; Chen et al. 2011). Be distinct from FSs and IFSs, FMSs, also known as bags, permit multiple occurrences of an element, and have wide applications in information retrieval (Miyamoto 2003). But, the basic operations of FMSs are not applied to FSs or IFSs. Furthermore, FSs, IFSs and FMSs all can be considered as particular cases of T2FSs. T2FSs, described by membership functions that are characterized by more parameters, permit the fuzzy membership as a FS improving the modeling capability of FSs (Hagras 2004; Doctor et al. 2005). However, T2FSs have some difficulties in establishing the secondary membership functions, and difficulties in manipulation (Karnik, Mendel 2001; Greenfield et al. 2009; Rickard et al. 2009).

HFSs, originally introduced by Torra (2010), have close relationships with IFSs and FMSs, and can also be considered as a particular case of T2FSs. The motivation to propose HFSs is that when defining the membership of an element, the difficulty of establishing the membership is not a margin of error (as in IFSs), or some possibility distributions (as in T2FSs) on the possible values, but a set of possible values. Torra (2010) gave an "membership problem" to illustrate this situation: two decision makers (DMs) discuss the membership of $x$ into $A$, and one wants to assign 0.5 and the other 0.6 , which can be denoted by a hesitant fuzzy element (HFE), $h=\{0.5,0.6\}$. In such a case, two values given by the two DMs for the membership can be collected into a single HFE.

Moreover, Zhu et al. (2012b) developed DHFSs as a new extension of HFSs. DHFSs are a comprehensive tool encompassing several existing fuzzy sets with certain conditions, whose membership and nonmembership are represented by a set of possible values respectively. For example, DHFSs permit the DMs consider as possible values for the membership a few different values as $0.1,0.2$ and 0.3 , and for the nonmembership as 0.4 and 0.5 . In particular cases, DHFSs can reduce to FSs, IFSs, HFSs or FMSs.

Comparing with FSs and IFSs, HFSs can be used to collect discrete data from the mathematical point of view. However, as the "membership problem" described above, if the two DMs both assign the value 0.5 , we can only save one value by the HFE, and loss the other one, which appears to be an information loss problem of HFSs. Further, since generallly the DMs have different importance in group decision making (Wei et al. 2012; Wu et al. 2012) due to their different social importance, position in the group, previous merits etc., a leading $\mathrm{DM}$ in a group for example, the loss of information provided by the leading DM may lead to ineffective results. Therefore, we should extend HFSs to overcome these limitations, which is also the topic we should articulate in this paper.

To resolve the information loss problem, we can collect the possible values provided by the DMs by several possible value-groups. For example, continued with the "membership problem", if one DM assigns 0.5 , another assigns 0.5 or 0.6 , we collect one membership provided by each DM together resulting in two possible value-groups as $(0.5,0.5)$ and $(0.5$, 0.6). In such a case, all the memberships provided by the DMs are saved and distinguished 
clearly in the value-groups. Motivated by this idea, we develop a concept of extended hesitant fuzzy sets (EHFSs) in this paper, which considers possible value-groups for the membership of $x$ into the set $A$. Furthermore, EHFSs have close relationships with IFSs, T2FSs, FMSs, DHFSs and especially HFSs. EHFSs are an extension of HFSs, on the contrary, HFSs can also be considered as a particular case of EHFSs. EHFSs increase the richness of numerical representation based on the value-groups, enhance the modeling abilities of HFSs, and can identify different DMs in decision making, which expand the applications of HFSs in practice.

On the other hand, the distance measure has received more and more attentions over the last decades and has wide applications in practice, such as pattern recognition (Li, Cheng 2002), cluster analysis (Yager 1988), approximate reasoning (Wang et al. 2004) and decision making (Wang, Xin 2005; Xu 2010). It is a significant research topic with respect to fuzzy theories. For example, some famous distance measures including Hamming distance, Euclidean distance and Hausdorff metric (Diamond, Kloeden 1994; Kacprzyk 1997; Chaudhuri, Rosenfeld 1999) have been extended to IFSs (Bustince, Burillo 1995; Szmidt, Kacprzyk 2000; $\mathrm{Xu}$ 2007b). For HFSs, Xu and Xia (2011) proposed some distance measures under the hesitant fuzzy environment, called hesitant distance measures, and gave some examples to show their applications in decision making.

As Xu and Xia (2011) explained, the hesitant distance measures can only be used in decision making with the conditions that all the DMs give their preferences anonymously or have the same importance so as to collect their preferences with no difference. This precondition interferes with the application of HFSs in practice, because the DMs usually have different importance in decision making. To deal with this problem, we develop some extended hesitant distance measures which take full advantages of EHFSs so as to show the advantages of HFESs comparing with HFSs. Furthermore, in order to take the DMs' knowledge (Yager et al. 1994) and risk preference (Liu, Wang 2007; Merigó, Casanovas 2009; Merigó, Gil-Lafuente 2009) into account, we combine the extended hesitant distance measures with Dempster-Shafer belief structure, and develop an approach to deal with group decision making problems. An energy policy example is also given to illustrate our results.

\section{Preliminaries}

Atanassov (1986) originally introduced the concept of intuitionistic fuzzy sets (IFSs) below.

Definition 1 (Atanassov, Gargov 1989). Let $X$ be a fixed set, an IFS $A$ on $X$ is represented in terms of two functions $\mu: X \rightarrow[0,1]$ and $v: X \rightarrow[0,1]$, with the condition, $0 \leq \mu(x)+v(x) \leq 1$, $\forall x \in X$, where $\mu$ represents the membership and $v$ the nonmembership of $\mathrm{x}$ into the set $\mathrm{A}$. IFSs are often represented as $\left\langle x, \mu_{A}, v_{A}\right\rangle$, for all $x \in X$.

For convenience, $\mathrm{Xu}$ and Yager $(2006)$ called $\left(\mu_{A}, v_{A}\right)$ an intuitionistic fuzzy number (IFN).

For three IFNs $\alpha, \alpha_{1}$ and $\alpha_{1}$, Xu (2007a) gave some operations on them, shown as follows:

1) $\alpha^{c}=\left(v_{\alpha}, \mu_{\alpha}\right)$;

2) $\alpha_{1} \oplus \alpha_{2}=\left(\mu_{\alpha_{1}}+\mu_{\alpha_{2}}-\mu_{\alpha_{1}} \mu_{\alpha_{2}}, v_{\alpha_{1}} v_{\alpha_{2}}\right)$;

3) $\alpha_{1} \otimes \alpha_{2}=\left(\mu_{\alpha_{1}} \mu_{\alpha_{2}}, v_{\alpha_{1}}+v_{\alpha_{2}}-v_{\alpha_{1}} v_{\alpha_{2}}\right)$; 
4) $\lambda \alpha=\left(1-\left(1-\mu_{\alpha}\right)^{\lambda}, v_{\alpha}^{\lambda}\right), \lambda>0$;

5) $\alpha^{\lambda}=\left(\mu_{\alpha}^{\lambda}, 1-\left(1-v_{\alpha}\right)^{\lambda}\right), \lambda>0$.

Torra (2010) defined hesitant fuzzy sets (HFSs) as follows.

Definition 2 (Torra 2010). Let $X$ be a fixed set, a HFS on $X$ is in terms of a function that when applied to $X$ returns a subset of $[0,1]$, which can be represented as the following mathematical symbol:

$$
E=\{<x, h(x)>\mid x \in X\},
$$

Where $h(x)$ is a finite set of some values in $[0,1]$, denoting the possible memberships of the element $x \in X$ to the set $E$.

For convenience, $h(x)$ is called a hesitant fuzzy element (HFE).

Given three HFEs $h, h_{1}$ and $h_{2}$, and let $h_{i}^{-}$and $h_{i}^{+}$be the minimum and maximum memberships in $h_{i} \quad(i=1,2)$ respectively, Torra (2010) defined some operations which can be represented as follows:

1) $h^{c}=\bigcup_{\gamma \in h}\{1-\gamma\}$;

2) $h_{1} \cup h_{2}=\left\{h \in\left(h_{1} \cup h_{2}\right) \mid h \geq \max \left(h_{1}^{-}, h_{2}^{-}\right)\right\}$;

3) $h_{1} \cap h_{2}=\left\{h \in\left(h_{1} \cap h_{2}\right) \mid h \leq \min \left(h_{1}^{+}, h_{2}^{+}\right)\right\}$.

$\mathrm{Xia}$ and $\mathrm{Xu}$ (2011) developed some new operations as below:

1) $h^{\lambda}=\bigcup_{\gamma \in h}\left\{\gamma^{\lambda}\right\}, \lambda>0$;

2) $\lambda h=\bigcup_{\gamma \in h}\left\{1-(1-\gamma)^{\lambda}\right\}, \lambda>0$;

3) $h_{1} \oplus h_{2}=\bigcup_{\gamma_{1} \in h_{1}, \gamma_{2} \in h_{2}}\left\{\gamma_{1}+\gamma_{2}-\gamma_{1} \gamma_{2}\right\}$;

4) $h_{1} \otimes h_{2}=\bigcup_{\gamma_{1} \in h_{1}, \gamma_{2} \in h_{2}}\left\{\gamma_{1} \gamma_{2}\right\}$.

Zhu et al. (2012a) further developed the following relationships for HFEs:

1) $\lambda\left(h_{1} \oplus h_{2}\right)=\lambda h_{1} \oplus \lambda h_{2}$;

2) $\left(h_{1} \otimes h_{2}\right)^{\lambda}=h_{1}^{\lambda} \otimes h_{2}^{\lambda}$.

$\mathrm{Xia}$ and $\mathrm{Xu}$ (2011) gave a method to rank any two HFEs as follows.

Definition 3 (Xia, Xu 2011). For a HFE $h, s(h)=(1 / \# h) \sum_{\gamma \in h} \gamma$ is called the score function of $h$, where $\# h$ is the number of the elements in $h$. Moreover, for two HFEs $h_{1}$ and $h_{2}$, if $s\left(h_{1}\right)>s\left(h_{2}\right)$, then $h_{1}>h_{2}$; if $s\left(h_{1}\right)=s\left(h_{2}\right)$, then $h_{1}=h_{2}$.

Torra (2010) gave a definition to the envelope of HFEs as follows.

Definition 4 (Torra 2010). Given a HFE $h$, an IFN $A_{e n v(h)}$ is defined as the envelope of $h$. This number, which will be denoted by $A_{\text {env }}(h)$, is represented by $(\mu, v)$ with $\mu$ and $v$ defined as $\mu=h^{-}$and $v=1-h^{+}$, where $h^{+}=\max \{\gamma \mid \gamma \in h\}$ and $h^{-}=\min \{\gamma \mid \gamma \in h\}$.

Furthermore, some properties of $A_{e n v}(h)$ are shown as follows:

1) $A_{\text {env }}\left(h^{c}\right)=\left(A_{\text {env }}(h)\right)^{c}$;

2) $A_{\text {env }}\left(h_{1} \cup h_{2}\right)=A_{\text {env }}\left(h_{1}\right) \cup A_{\text {env }}\left(h_{2}\right)$;

3) $A_{\text {env }}\left(h_{1} \cap h_{2}\right)=A_{\text {env }}\left(h_{1}\right) \cap A_{\text {env }}\left(h_{2}\right)$.

Zhu et al. (2012b) originally introduced the concept of dual hesitant fuzzy sets (DHFSs). 
Definition 5 (Zhu et al. 2012b). Let X be a fixed set, then a DHFS D on X is described as:

$$
D=\{<x, h(x), g(x)>\mid x \in X\},
$$

in which $h(x)$ and $g(x)$ are two sets of some values in $[0,1]$, denoting the possible memberships and nonmemberships of the element $x \in X$ to the set $D$ respectively, with the conditions $0 \leq \gamma, \eta \leq 1,0 \leq \gamma^{+}+\eta^{+} \leq 1$, where $\gamma \in h(x), \eta \in g(x), \gamma^{+}=\bigcup_{\gamma \in h(x)} \max \{\gamma\}$, and $\eta^{+}=\bigcup_{\eta \in g(x)} \max \{\eta\}$ for each $x \in X$.

For convenience, the pair $d(x)=(h(x), g(x))$ is called a dual hesitant fuzzy element (DHFE) denoted by $d=(h, g)$.

\section{EHFSs and basic operations and properties}

\subsection{EHFSs}

Given several HFSs, we use a Cartesian product of HFSs to construct an extended hesitant fuzzy set (EHFS) as follows.

Definition 6. Let $X$ be a fixed set, $h_{D}(x)=\bigcup_{\gamma_{D} \in h_{D}(x)}\left\{\gamma_{D}\right\} \quad(D=1, \ldots, m)$ be HFSs on $X$. Then, an EHFS, that is $H_{h_{D}}$, is defined as:

$$
H_{h_{D}}(x)=h_{1}(x) \times \ldots \times h_{m}(x)=\bigcup_{\gamma_{1} \in h_{1}(x), \ldots, \gamma_{m} \in h_{m}(x)}\left\{<x,\left(\gamma_{1}(x), \ldots, \gamma_{m}(x)\right)>\mid x \in X\right\} \text {. }
$$

For convenience, we call:

$$
H=h_{1} \times \ldots \times h_{m}=\bigcup_{\gamma_{1} \in h_{1}, \ldots, \gamma_{m} \in h_{m}}\left\{\left(\gamma_{1}, \ldots, \gamma_{m}\right)\right\}
$$

an extended hesitant fuzzy element (EHFE).

For $H=\bigcup_{\gamma_{1} \in h_{1}, \ldots, \gamma_{m} \in h_{m}}\left\{\left(\gamma_{1}, \ldots, \gamma_{m}\right)\right\}$, let $u=\left(\gamma_{1}, \ldots, \gamma_{m}\right)$, then we call $\mathrm{u}$ a membership unit (MU). Based on $\mathrm{u}$, an EHFE $H$, can also be indicated by:

$$
H=\bigcup_{u \in H}\{u\}=\bigcup_{\left(\gamma_{1}, \ldots, \gamma_{m}\right) \in H}\left\{\left(\gamma_{1}, \ldots, \gamma_{m}\right)\right\} .
$$

HFSs can be used to construct EHFSs. On the contrary, EHFSs can reduce to HFSs. To investigate the relationship between HFSs and EHFSs, we develop a concept of reduced EHFEs.

Definition 7. Given an EHFE $H=\bigcup_{\left(\gamma_{1}, \ldots, \gamma_{m}\right) \in H}\left\{\left(\gamma_{1}, \ldots, \gamma_{m}\right)\right\}$, then

$$
h_{H}=\bigcup_{\left(\gamma_{1}, \ldots, \gamma_{m}\right) \in H}\left\{\gamma_{1}, \ldots, \gamma_{m}\right\}=\bigcup_{\gamma \in H}\{\gamma\}
$$

is called a reduced EHFE.

Furthermore, if there is only one MU in $H$, i.e., $H=\left\{\left(\gamma_{1}, \ldots, \gamma_{m}\right)\right\}$ and satisfying $\left(\gamma_{1} \neq, \ldots, \neq \gamma_{m}\right)$, we consider that the EHFE $H$ is equivalent to a HFE, denoted by $h_{H}=\left\{\gamma_{1}, \ldots, \gamma_{m}\right\}$.

Proposition 1. The HFS $h(x)$ is a particular case of the EHFS $H(x)$, where $H(x)=\left\{\left(\gamma_{1}(x), \ldots, \gamma_{m}(x)\right)\right\} \quad\left(\gamma_{1}(x) \neq, \ldots, \neq \gamma_{m}(x)\right)$, for each element in the domain.

Consider that IFSs are a particular case of HFSs, where HFSs are nonempty closed intervals (Torra 2010). By an operation of envelope for EHFSs defined in the rest of the paper, we can also transform EHFSs into closed intervals. Therefore, IFSs can also be considered as a particular case of EHFSs. We state this below. 
Proposition 2. IFSs are a particular case of EHFSs, where EHFSs are nonempty closed intervals.

Moreover, as discussed by Zhu et al. (2012b), for a given DHFS $d(x) \neq \varnothing$, if $h(x)$ and $g(x)$ have only one value for each element in the domain, then DHFSs reduce to IFSs; if $g(x)=\varnothing$ and $h(x) \neq \varnothing$, then DHFSs reduce to HFSs. Thus, with the analyses above and according to Propositions 1 and 2, we conclude that DHFSs can also be considered as a particular case of EHFSs when DHFSs reduce to IFSs or HFSs.

\subsection{Basic operations and properties}

Definition 8. Let $H=\bigcup_{\gamma_{1} \in h_{1}, \ldots, \gamma_{m} \in h_{m}}\left\{\left(\gamma_{1}, \ldots, \gamma_{m}\right)\right\}$ be an EHFE, then we define its complement as:

$$
H^{c}=\bigcup_{\left(\gamma_{1}, \ldots, \gamma_{m}\right) \in H}\left\{\left(1-\gamma_{1}, \ldots, 1-\gamma_{m}\right)\right\}
$$

Since each $u$ can be considered as a HFE, by the operation of HFEs, Eq.(7) can also be denoted as:

$$
H^{c}=\bigcup_{u \in H}\left\{u^{c}\right\} .
$$

Obviously, the complement of complement of an EHFE is itself, which can be concluded as below.

Proposition 3. The complement of an EHFE is involutive, denoted by $\left(H^{c}\right)^{c}=H$.

Given an EHFE $H=\bigcup_{u \in H}\{u\}$, we define the minimum and maximum memberships of $H$ as follows:

1) The minimum membership of $H: \gamma_{H}^{-}=\min \left\{\gamma \mid \gamma \in h_{H}\right\}$;

2) The maximum membership of $H: \gamma_{H}^{+}=\max \left\{\gamma \mid \gamma \in h_{H}\right\}$.

where $h_{H}$ is the reduced EHFE of $H$.

Further, the minimum and maximum memberships of $u$ can be defined as follows:

1) The minimum membership of $u: u^{-}=\min \{\gamma \mid \gamma \in u\}$;

2) The maximum membership of $u: u^{+}=\max \{\gamma \mid \gamma \in u\}$.

For any two EHFEs, $H_{1}$ and $H_{2}$, we now define their union and intersection.

Definition 9. Given two EHFEs, $H_{1}$ and $H_{2}$, the union of them is defined as:

$$
H_{1} \cup H_{2}=\bigcup_{u \in\left(H_{1} \cup H_{2}\right)}\left\{u \mid u^{-} \geq \max \left(\gamma_{H_{1}}^{-}, \gamma_{H_{2}}^{-}\right)\right\} \text {; }
$$

or equivalently:

$$
H_{1} \cup H_{2}=\bigcup_{u_{1} \in H_{1}, u_{2} \in H_{2}}\left\{u_{1}, u_{2} \mid u_{1}^{-}, u_{2}^{-} \geq \max \left(\gamma_{H_{1}}^{-}, \gamma_{H_{2}}^{-}\right)\right\}
$$

The intersection of them is defined as:

$$
H_{1} \cap H_{2}=\bigcup_{u \in\left(H_{1} \cap H_{2}\right)}\left\{u \mid u^{+} \leq \min \left(\gamma_{H_{1}}^{+}, \gamma_{H_{2}}^{+}\right)\right\} ;
$$

or equivalently:

$$
H_{1} \cap H_{2}=\bigcup_{u_{1} \in H_{1}, u_{2} \in H_{2}}\left\{u_{1}, u_{2} \mid u_{1}^{+}, u_{2}^{+} \leq \min \left(\gamma_{H_{1}}^{+}, \gamma_{H_{2}}^{+}\right)\right\}
$$

where $\gamma_{H_{1}}^{+}$and $\gamma_{H_{2}}^{+}$are the maximum memberships in $H_{1}$ and $H_{2}$ respectively. 
Example 1. Let $H_{1}=\{(0.2,0.3),(0.2,0.4)\}$ and $H_{2}=\{(0.3,0.4)\}$ be two EHFEs, we have $\gamma_{H_{1}}^{-}=0.2, \gamma_{H_{2}}^{-}=0.3, \gamma_{H_{1}}^{+}=0.4$ and $\gamma_{H_{2}}^{+}=0.4$. By Definition 9, we can get:

$$
H_{1} \cup H_{2}=\{(0.3,0.4)\}, H_{1} \cap H_{2}=\{(0.2,0.3),(0.2,0.4),(0.3,0.4)\} .
$$

The operations between EHFEs and HFEs have a close relationship.

Proposition 4. Assume two EHFEs, $H_{1}$ and $H_{2}$, and two reduced EHFEs of $H_{1}$ and $H_{2}, h_{H_{1}}$ and $h_{H_{2}}$, the following are valid:

1) $h_{\left(H_{1} \cup H_{2}\right)}=h_{H_{1}} \cup h_{H_{2}}$;

2) $h_{\left(H_{1} \cap H_{2}\right)}=h_{H_{1}} \cap h_{H_{2}}$.

\section{Proof.}

1) For any two EHFEs, $H_{1}$ and $H_{2}$, by the operation of HFEs and Eq. (6), we can get:

$$
h_{H_{1}} \cup h_{H_{2}}=\bigcup_{\gamma_{1} \in h_{H_{1}}, \gamma_{2} \in h_{H_{2}}}\left\{\gamma_{1}, \gamma_{2} \mid \gamma_{1}, \gamma_{2} \geq \max \left(\gamma_{H_{1}}^{-}, \gamma_{H_{2}}^{-}\right)\right\} \text {. }
$$

By Eq. (6), it can be shown that:

Since:

$$
h_{\left(H_{1} \cup H_{2}\right)}=\bigcup_{\gamma \in\left(h_{H_{1}} \cup h_{H_{2}}\right)}\{\gamma\} .
$$

$$
H_{1} \cup H_{2}=\bigcup_{u \in\left(H_{1} \cup H_{2}\right)}\left\{u \mid u^{-} \geq \max \left(\gamma_{H_{1}}^{-}, \gamma_{H_{2}}^{-}\right)\right\}
$$

then:

$$
\begin{aligned}
& h_{\left(H_{1} \cup H_{2}\right)}=\bigcup_{\gamma \in u}\left\{\gamma \mid u \in\left(H_{1} \cup H_{2}\right), u^{-} \geq \max \left(\gamma_{H_{1}}^{-}, \gamma_{H_{2}}^{-}\right)\right\}= \\
& \bigcup_{\gamma_{1} \in h_{H_{1}}, \gamma_{2} \in h_{H_{2}}}\left\{\gamma_{1}, \gamma_{2} \mid \gamma_{1}, \gamma_{2} \in u, u \in\left(H_{1} \cup H_{2}\right), u^{-} \geq \max \left(\gamma_{H_{1}}^{-}, \gamma_{H_{2}}^{-}\right)\right\}= \\
& \bigcup_{\gamma_{1} \in h_{H_{1}}, \gamma_{2} \in h_{H_{1}}}\left\{\gamma_{1}, \gamma_{2} \mid \gamma_{1}, \gamma_{2} \geq \max \left(\gamma_{H_{1}}^{-}, \gamma_{H_{2}}^{-}\right)\right\}= \\
& h_{H_{1}} \cup h_{H_{2}},
\end{aligned}
$$

which completes the proof.

The proof of the intersection of EHFEs is similar to that of the proof of union above, which is not listed here.

HFEs and IFNs have a close relationship that HFEs are deemed IFNs when HFEs are nonempty closed intervals. Given an IFN, $(\mu, v)$, we can get a corresponding HFE, $h$, denoted by an interval $h=[\mu, 1-v]$ if $\mu \neq 1-v$; given a HFE, $h$, the envelope of $h$ is an IFN, i.e., $A_{\text {env }}(h)=\left(h^{-}, 1-h^{+}\right)$. The envelope of EHFEs also has close connections with HFEs and IFNs. We now give a definition of the envelope of EHFEs.

Definition 10. Given an EHFE $H=\bigcup_{u \in H}\{u\}$, the envelope of $H$ can be defined as $A_{\text {env }}(H)=\bigcup_{\mu \in \mathcal{u}^{-}, v \in \mathcal{u}^{+}}\{(\mu, v) \mid u \in H\}$, where $\mathcal{u}^{-}$and $u^{+}$are the minimum and maximum memberships of $\mathrm{u}$, respectively.

It's clear that the envelope of an EHFE may include several IFNs. In addition, in the particular case that an EHFE, $H$, is equivalent to a HFE, $h$ (proposed in Proposition 1 ), 
the envelope of $H$ is equivalent to the envelope of h, i.e., $A_{\text {env }}(H)=A_{\text {env }}(h)$. Thus, $A_{\text {env }}(h)$ is also a particular case of $A_{\text {env }}(H)$, which is stated as below.

Proposition 5. $A_{\text {env }}(h)$ is a particular case of $A_{e n v}(H)$, when $H$ is equivalent to $h$.

We can further propose a proposition of $A_{\text {env }}(H)$ below.

Proposition 6. For an EHFEs $H$ and its envelope $A_{\text {env }}(H)$, we have $A_{\text {env }}\left(H^{c}\right)=\left(A_{\text {env }}(H)\right)^{c}$.

Proof. For an EHFE $H$, and its envelope $A_{\text {env }}(H)$, since:

$$
\begin{aligned}
& A_{\text {env }}\left(H^{c}\right)=A_{\text {env }}\left(\bigcup_{u \in H}\{u\}\right)=\bigcup_{u^{+}, u^{-} \in u^{\prime}}\left\{\left(1-u^{+}, 1-1+u^{-}\right) \mid u \in H\right\}= \\
& \bigcup_{u^{+}, u^{-} \in u}\left\{\left(1-u^{+}, u^{-}\right) \mid u \in H\right\}
\end{aligned}
$$

and

$$
\left(A_{\text {env }}(H)\right)^{c}=\bigcup_{u^{+}, u^{-} \in u^{-}}\left\{\left(u^{-}, 1-u^{+}\right)^{c} \mid u \in H\right\}=\bigcup_{u^{+}, u^{-} \in u^{-}}\left\{\left(1-u^{+}, u^{-}\right) \mid u \in H\right\},
$$

then $A_{\text {env }}\left(H^{c}\right)=\left(A_{\text {env }}(H)\right)^{c}$, which completes the proof.

We now develop some operations of EHFEs further.

Definition 11. Given three EHFEs, $H=\bigcup_{u \in H}\{u\}, H_{1}=\bigcup_{u_{1} \in H_{1}}\left\{u_{1}\right\}, H_{2}=\bigcup_{u_{2} \in H_{2}}\left\{u_{2}\right\}$, $\lambda>0$, since the MUs $u, u_{1}$ and $u_{2}$ can be considered as three HFEs, we have the following operations:

1) $H^{\lambda}=\bigcup_{u \in H}\left\{u^{\lambda}\right\}$

2) $\lambda H=\bigcup_{u \in H}\{\lambda u\}$;

3) $H_{1} \oplus H_{2}=\bigcup_{u_{1} \in H_{1}, u_{2} \in H_{2}}\left\{u_{1} \oplus u_{2}\right\}$;

4) $H_{1} \otimes H_{2}=\bigcup_{u_{1} \in H_{1}, u_{2} \in H_{2}}\left\{u_{1} \otimes u_{2}\right\}$.

Based on Definition 11, we can prove the following proposition.

Proposition 7. For two EHFEs, $H_{1}$ and $H_{2}, \lambda>0$, we have:

1) $H_{1} \oplus H_{2}=H_{2} \oplus H_{1}$;

2) $H_{1} \otimes H_{2}=H_{2} \otimes H_{1}$;

3) $\lambda\left(H_{1} \oplus H_{2}\right)=\lambda H_{1} \oplus \lambda H_{2}$;

4) $\left(H_{1} \otimes H_{2}\right)^{\lambda}=H_{1}^{\lambda} \otimes H_{2}^{\lambda}$.

Proof. For any two EHFEs, $H_{1}$ and $H_{2}, \lambda>0$, based on the operations and relationships of HFEs, we can get:

1) $H_{1} \oplus H_{2}=\bigcup_{u_{1} \in H_{1}, u_{2} \in H_{2}}\left\{u_{1} \oplus u_{2}\right\}=\bigcup_{u_{1} \in H_{1}, u_{2} \in H_{2}}\left\{u_{2} \oplus u_{1}\right\}=H_{2} \oplus H_{1}$;

2) $H_{1} \otimes H_{2}=\bigcup_{u_{1} \in H_{1}, u_{2} \in H_{2}}\left\{u_{1} \otimes u_{2}\right\}=\bigcup_{u_{1} \in H_{1}, u_{2} \in H_{2}}\left\{u_{2} \otimes u_{1}\right\}=H_{2} \otimes H_{1}$;

3) $\lambda\left(H_{1} \oplus H_{2}\right)=\bigcup_{u_{1} \in H_{1}, u_{2} \in H_{2}}\left\{\lambda\left(u_{1} \oplus u_{2}\right)\right\}=\bigcup_{u_{1} \in H_{1}, u_{2} \in H_{2}}\left\{\lambda u_{1} \oplus \lambda u_{2}\right\}=$ $\bigcup_{u_{1} \in H_{1}}\left\{\lambda u_{1}\right\} \oplus \bigcup_{u_{2} \in H_{2}}\left\{\lambda u_{2}\right\}=\lambda H_{1} \oplus \lambda H_{2} ;$

4) $\left(H_{1} \otimes H_{2}\right)^{\lambda}=\bigcup_{u_{1} \in H_{1}, u_{2} \in H_{2}}\left\{\left(u_{1} \otimes u_{2}\right)^{\lambda}\right\}=\bigcup_{u_{1} \in H_{1}, u_{2} \in H_{2}}\left\{u_{1}^{\lambda} \otimes u_{2}{ }^{\lambda}\right\}=H_{1}{ }^{\lambda} \otimes H_{2}{ }^{\lambda}$. 
Proposition 8. For any three EHFEs $H, H_{1}$ and $H_{2}$, and their reduced EHFEs $h_{H}, h_{H_{1}}$ and $h_{\mathrm{H}_{2}}, \lambda>0$, the following are valid:

1) $h_{H^{\lambda}}=\left(h_{H}\right)^{\lambda}$;

2) $h_{\lambda H}=\lambda\left(h_{H}\right)$;

3) $h_{\left(H_{1} \oplus H_{2}\right)}=h_{H_{1}} \oplus h_{H_{2}}$;

4) $h_{\left(H_{1} \otimes H_{2}\right)}=h_{H_{1}} \otimes h_{H_{2}}$.

Proof. For any three EHFEs $H, H_{1}$ and $H_{2}$, and their reduced EHFEs $h_{H}, h_{H_{1}}$ and $h_{\mathrm{H}_{2}}, \lambda>0$, we have:

1) $h_{H^{\lambda}}=\bigcup_{\gamma \in u}\left\{\gamma^{\lambda} \mid u \in H\right\}=\bigcup_{\gamma \in h_{H}}\left\{\gamma^{\lambda}\right\}=\left(h_{H}\right)^{\lambda}$;

2) $h_{\lambda H}=\bigcup_{\gamma \in u}\left\{1-(1-\gamma)^{\lambda} \mid u \in H\right\}=\bigcup_{\gamma \in h_{H}}\left\{1-(1-\gamma)^{\lambda}\right\}=\lambda\left(h_{H}\right)$;

3) $h_{\left(H_{1} \oplus H_{2}\right)}=\bigcup_{\gamma_{1} \in u_{1}, \gamma_{2} \in u_{2}}\left\{\gamma_{1}+\gamma_{2}-\gamma_{1} \gamma_{2} \mid u_{1} \in H_{1}, u_{2} \in H_{2}\right\}=$

$\bigcup_{\gamma_{1} \in h_{H_{1}}, \gamma_{2} \in h_{H_{2}}}\left\{\gamma_{1}+\gamma_{2}-\gamma_{1} \gamma_{2}\right\}=h_{H_{1}} \oplus h_{H_{2}}$

4) $h_{\left(H_{1} \otimes H_{2}\right)}=\bigcup_{\gamma_{1} \in u_{1}, \gamma_{2} \in u_{2}}\left\{\gamma_{1} \gamma_{2} \mid u_{1} \in H_{1}, u_{2} \in H_{2}\right\}=\bigcup_{\gamma_{1} \in h_{H_{1}}, \gamma_{2} \in h_{H_{2}}}\left\{\gamma_{1} \gamma_{2}\right\}=h_{H_{1}} \otimes h_{H_{2}}$.

Proposition 9. For any three EHFEs $H, H_{1}$ and $H_{2}, \lambda>0$, we have:

1) $A_{\text {env }}\left(H^{\lambda}\right)=\left(A_{\text {env }}(H)\right)^{\lambda}$;

2) $A_{\text {env }}(\lambda H)=\lambda\left(A_{\text {env }}(H)\right)$;

3) $A_{\text {env }}\left(H_{1} \oplus H_{2}\right)=A_{\text {env }}\left(H_{1}\right) \oplus A_{\text {env }}\left(H_{2}\right)$;

4) $A_{\text {env }}\left(H_{1} \otimes H_{2}\right)=A_{\text {env }}\left(H_{1}\right) \otimes A_{e n v}\left(H_{2}\right)$.

Proof. For any three EHFEs $H, H_{1}$ and $H_{2}, \lambda>0$, we have:

1) $A_{\text {env }}\left(H^{\lambda}\right)=A_{\text {env }}\left(\bigcup_{u \in H}\left\{u^{\lambda}\right\}\right)=$

$\bigcup_{u^{-}, u^{+} \in u^{-}}\left\{\left(\left(u^{-}\right)^{\lambda}, 1-\left(u^{+}\right)^{\lambda}\right) \mid u \in H\right\}=$

$\bigcup_{u^{-}, u^{+} \in u}\left\{\left(\left(u^{-}\right)^{\lambda}, 1-\left(1-\left(1-u^{+}\right)\right)^{\lambda}\right) \mid u \in H\right\}=$

$\bigcup_{u^{-}, u^{+} \in u^{\prime}}\left\{\left(\left(u^{-}, 1-u^{+}\right)^{\lambda}\right) \mid u \in H\right\}=\left(A_{\text {env }}(H)\right)^{\lambda}=$;

2) $A_{\text {env }}(\lambda H)=A_{\text {env }}\left(\bigcup_{u \in H}\{\lambda u)\right)=$

$\bigcup_{u^{-}, u^{+} \in u^{-}}\left\{\left(1-\left(1-u^{-}\right)^{\lambda}, 1-\left(1-\left(1-u^{+}\right)^{\lambda}\right)\right) \mid u \in H\right\}=$

$\left.\bigcup_{u^{-}, u^{+} \in u}\left\{\left(1-\left(1-u^{-}\right)^{\lambda},\left(1-u^{+}\right)^{\lambda}\right)\right) \mid u \in H\right\}=$

$\lambda\left(\bigcup_{u^{-}, u^{+} \in u^{\prime}}\left\{\left(u^{-}, 1-u^{+}\right) \mid u \in H\right\}\right)=$

$\lambda\left(A_{\text {env }}(H)\right)$; 
3) $A_{\text {env }}\left(H_{1} \oplus H_{2}\right)=A_{\text {env }}\left(\bigcup_{u_{1} \in H_{1}, u_{2} \in H_{2}}\left\{u_{1} \oplus u_{2}\right)=\right.$

$\bigcup_{u_{1}^{-}, u_{1}^{+} \in u_{1}, u_{2}^{-}, u_{2}^{+} \in u_{2}}\left\{\left(u_{1}^{-}+u_{2}^{-}-u_{1}^{-} u_{2}^{-}, 1-\left(u_{1}^{+}+u_{2}^{+}-u_{1}^{+} u_{2}^{+}\right)\right) \mid u_{1} \in H_{1}, u_{2} \in H_{2}\right\}=$

$\bigcup_{u_{1}^{-}, u_{1}^{+} \in u_{1}, u_{2}^{-}, u_{2}^{+} \in u_{2}}\left\{\left(u_{1}^{-}+u_{2}^{-}-u_{1}^{-} u_{2}^{-},\left(1-u_{1}^{+}\right)\left(1-u_{2}^{+}\right)\right) \mid u_{1} \in H_{1}, u_{2} \in H_{2}\right\}=$

$\left(\bigcup_{u_{1}^{-}, u_{1}^{+} \in u_{1}}\left\{\left(u_{1}^{-}, 1-u_{1}^{+}\right) \mid u_{1} \in H_{1}\right\}\right) \oplus\left(\bigcup_{u_{2}^{-}, u_{2}^{+} \in u_{2}}\left\{\left(u_{2}^{-}, 1-u_{2}^{+}\right) \mid u_{2} \in H_{2}\right\}\right)=$

$A_{\text {env }}\left(H_{1}\right) \oplus A_{\text {env }}\left(H_{2}\right)$;

4) $A_{\text {env }}\left(H_{1} \otimes H_{2}\right)=A_{e n v}\left(H_{1} \otimes H_{2}\right)=A_{e n v}\left(\bigcup_{u_{1} \in H_{1}, u_{2} \in H_{2}}\left\{u_{1} \otimes u_{2} \mid u_{1} \in H_{1}, u_{2} \in H_{2}\right\}\right)=$

$\bigcup_{u_{1}^{-}, u_{1}^{+} \in u_{1}, u_{2}^{-}, u_{2}^{+} \in u_{2}}\left\{\left(u_{1}^{-} u_{2}^{-}, 1-u_{1}^{+} u_{2}^{+}\right) \mid u_{1} \in H_{1}, u_{2} \in H_{2}\right\}=$

$\bigcup_{u_{1}^{-}, u_{1}^{+} \in u_{1}, u_{2}^{-}, u_{2}^{+} \in u_{2}}\left\{\left(\left(u_{1}^{-} u_{2}^{-},\left(1-u_{1}^{+}\right)+\left(1-u_{2}^{+}\right)-\left(1-u_{1}^{+}\right)\left(1-u_{2}^{+}\right)\right)\right) \mid u_{1} \in H_{1}, u_{2} \in H_{2}\right\}=$

$\bigcup_{u_{1}^{-}, u_{1}^{+} \in u_{1}}\left\{\left(u_{1}^{-}, 1-u_{1}^{+}\right) \mid u_{1} \in H_{1}\right\} \oplus \bigcup_{u_{2}^{-}, u_{2}^{+} \in u_{2}}\left\{\left(u_{2}^{-}, 1-u_{2}^{+}\right) \mid u_{2} \in H_{2}\right\}=$

$A_{\text {env }}\left(H_{1}\right) \otimes A_{\text {env }}\left(H_{2}\right)$.

Proposition 10. For any three EHFEs $H, H_{1}$ and $H_{2}, \lambda>0$, we have:

1) $H_{1}^{c} \cup H_{2}^{c}=\left(H_{1} \cap H_{2}\right)^{c}$;

2) $H_{1}^{c} \cap H_{2}^{c}=\left(H_{1} \cup H_{2}\right)^{c}$;

3) $\lambda\left(H^{c}\right)=\left(H^{\lambda}\right)^{c}$;

4) $\left(H^{c}\right)^{\lambda}=(\lambda H)^{c}$;

5) $H_{1}^{c} \oplus H_{2}^{c}=\left(H_{1} \otimes H_{2}\right)^{c}$;

6) $H_{1}^{c} \otimes H_{2}^{c}=\left(H_{1} \oplus H_{2}\right)^{c}$.

Proof. For any three EHFEs $H, H_{1}$ and $H_{2}, \lambda>0$, we have:

1) $H_{1}^{c} \cup H_{2}^{c}=\bigcup_{u_{1} \in H_{1}, u_{2} \in H_{2}}\left\{u_{1}^{c}, u_{2}^{c} \mid\left(u_{1}^{c}\right)^{-},\left(u_{2}^{c}\right)^{-} \geq \max \left(\left(1-\gamma_{H_{1}}^{+}\right),\left(1-\gamma_{H_{2}}^{+}\right)\right\}=\right.$

$\bigcup_{u_{1} \in H_{1}, u_{2} \in H_{2}}\left\{u_{1}^{c}, u_{2}^{c} \mid\left(u_{1}^{c}\right)^{-},\left(u_{2}^{c}\right)^{-} \geq 1-\min \left(\gamma_{H_{1}}^{+}, \gamma_{H_{2}}^{+}\right)\right\}=$

$\left(\bigcup_{u_{1} \in H_{1}, u_{2} \in H_{2}}\left\{u_{1}, u_{2} \mid u_{1}^{+}, u_{2}^{+} \leq \min \left(\gamma_{H_{1}}^{+}, \gamma_{H_{2}}^{+}\right)\right\}\right)^{c}=$

$\left(H_{1} \cap H_{2}\right)^{c}$;

2) $H_{1}^{c} \cap H_{2}^{c}=\bigcup_{u_{1} \in H_{1}, u_{2} \in H_{2}}\left\{u_{1}^{c}, u_{2}^{c} \mid\left(u_{1}^{c}\right)^{+},\left(u_{2}^{c}\right)^{+} \leq \min \left(\left(1-\gamma_{H_{1}}^{-}\right),\left(1-\gamma_{H_{2}}^{-}\right)\right)\right\}=$

$\bigcup_{u_{1} \in H_{1}, u_{2} \in H_{2}}\left\{u_{1}^{c}, u_{2}^{c} \mid\left(u_{1}^{c}\right)^{+},\left(u_{2}^{c}\right)^{+} \leq 1-\max \left(\gamma_{H_{1}}^{-}, \gamma_{H_{2}}^{-}\right)\right\}=$

$\left(\bigcup_{u_{1} \in H_{1}, u_{2} \in H_{2}}\left\{u_{1}, u_{2} \mid u_{1}^{-}, u_{2}^{-} \geq \max \left(\gamma_{H_{1}}^{-}, \gamma_{H_{2}}^{-}\right)\right\}\right)^{c}=$

$\left(H_{1} \cup H_{2}\right)^{c}$

3) $\lambda\left(H^{c}\right)=\bigcup_{u \in H}\left\{\lambda\left(u^{c}\right)\right\}=\bigcup_{\left(\gamma_{1}, \ldots, \gamma_{m}\right) \in H}\left\{\left(1-\left(1-\left(1-\gamma_{1}\right)\right)^{\lambda}, \ldots, 1-\left(1-\left(1-\gamma_{m}\right)\right)^{\lambda}\right)\right\}=$

$\bigcup_{\left(\gamma_{1}, \ldots, \gamma_{m}\right) \in H}\left\{\left(\left(1-\gamma_{1}\right)^{\lambda}, \ldots,\left(1-\gamma_{m}\right)^{\lambda}\right)\right\}=\bigcup_{u \in H}\left\{\left(u^{\lambda}\right)^{c}\right\}=\left(H^{\lambda}\right)^{c}$ 
4) $\left(H^{c}\right)^{\lambda}=\bigcup_{u \in H}\left\{\left(u^{c}\right)^{\lambda}\right\}=\bigcup_{\left(\gamma_{1}, \ldots, \gamma_{m}\right) \in H}\left\{\left(\left(1-\gamma_{1}\right)^{\lambda}, \ldots,\left(1-\gamma_{m}\right)^{\lambda}\right)\right\}=$

$$
\begin{aligned}
& \bigcup_{\left(\gamma_{1}, \ldots, \gamma_{m}\right) \in H}\left\{\left(\left(1-\left(1-\gamma_{1}\right)^{\lambda}\right)^{c}, \ldots,\left(1-\left(1-\gamma_{m}\right)^{\lambda}\right)^{c}\right)\right\}= \\
& \bigcup_{u \in H}\left\{(\lambda u)^{c}\right\}=(\lambda H)^{c} ;
\end{aligned}
$$

5) Since $u_{1}^{c} \oplus u_{2}^{c}=\bigcup_{\gamma_{1} \in u_{1}, \gamma_{2} \in u_{2}}\left\{\left(1-\gamma_{1}\right)+\left(1-\gamma_{2}\right)-\left(1-\gamma_{1}\right)\left(1-\gamma_{2}\right)\right\}=$

$$
\bigcup_{\gamma_{1} \in u_{1}, \gamma_{2} \in u_{2}}\left\{1-\gamma_{1} \gamma_{2}\right\}=\left(u_{1} \otimes u_{2}\right)^{c},
$$

we can get $H_{1}^{c} \oplus H_{2}^{c}=\bigcup_{u_{1} \in H_{1}, u_{2} \in H_{2}}\left\{u_{1}^{c} \oplus u_{2}^{c}\right\}=\bigcup_{u_{1} \in H_{1}, u_{2} \in H_{2}}\left\{\left(u_{1} \otimes u_{2}\right)^{c}\right\}=\left(H_{1} \otimes H_{2}\right)^{c}$;

6) Since $u_{1}^{c} \otimes u_{2}^{c}=\bigcup_{\gamma_{1} \in u_{1}, \gamma_{2} \in u_{2}}\left\{\left(1-\gamma_{1}\right)\left(1-\gamma_{2}\right)\right\}=\bigcup_{\gamma_{1} \in u_{1}, \gamma_{2} \in u_{2}}\left\{\left(1-\gamma_{1}-\gamma_{2}+\gamma_{1} \gamma_{2}\right)\right\}=$

$$
\bigcup_{\gamma_{1} \in u_{1}, \gamma_{2} \in u_{2}}\left\{1-\left(\gamma_{1}+\gamma_{2}-\gamma_{1} \gamma_{2}\right)\right\}=\left(u_{1} \oplus u_{2}\right)^{c},
$$

then $H_{1}^{c} \otimes H_{2}^{c}=\bigcup_{u_{1} \in H_{1}, u_{2} \in H_{2}}\left\{u^{c} \otimes u^{c}\right\}=\bigcup_{u_{1} \in H_{1}, u_{2} \in H_{2}}\left\{\left(u_{1} \oplus u_{2}\right)^{c}\right\}=\left(H_{1} \oplus H_{2}\right)^{c}$.

For a given EHFS $H$ on $X$, we have $H(x)$ for all $x$ in $X$. Then, we can define the EHFS as a fuzzy multiset (FMS) as:

$$
F M S_{H}=\oplus_{x \in X} \oplus_{\gamma \in u}\{(x, \gamma) \mid u \in H(x)\} .
$$

Thus, we can give the relationship between EHFSs and FMSs below.

Proposition 11. EHFSs can be represented as FMSs.

Similar to HFSs, the operations for FMSs also do not apply correctly to the EHFSs. Given an EHFS $H$ on $X$, for all $x$ in $X$, we can also define the EHFS as the following type- 2 fuzzy set (T2FS):

$$
\mu_{H(x)}(\gamma)=\left\{\begin{array}{l}
1, \gamma \in \mathcal{u}, u \in H(x) \\
0, \gamma \notin \mathcal{u}, u \in H(x)
\end{array}(x \in X) .\right.
$$

Thus, we can derive the following result.

Proposition 12. EHFSs can be represented as T2FSs.

\section{Extended hesitant distance measures}

In the following, we put forward the axioms of extended hesitant distance measures.

Definition 12. Let $H_{1}$ and $H_{2}$ be two EHFEs, then the extended hesitant distance measure is denoted by $d\left(H_{1}, H_{2}\right)$, which satisfies the following properties:

1) $0 \leq d\left(H_{1}, H_{2}\right) \leq 1$;

2) $d\left(H_{1}, H_{2}\right)=0$ if and only if $H_{1}=H_{2}$;

3) $d\left(H_{1}, H_{2}\right)=d\left(H_{2}, H_{1}\right)$.

For any finite universe set $X=\left\{x_{1}, \ldots, x_{n}\right\}$, Bustince and Burillo (1995) defined some distance measures between two IFSs $A_{1}(x)$ and $A_{2}(x)$ on $X$. For two IFNs, $A_{1}$ and $A_{2}$, we have:

1) The normalized Hamming distance

$$
d_{1}\left(A_{1}, A_{2}\right)=\frac{1}{2}\left(\left|\mu_{A_{1}}-\mu_{A_{2}}\right|+\left|v_{A_{1}}-v_{A_{2}}\right|\right) ;
$$


2) The normalized Euclidean distance

$$
d_{2}\left(A_{1}, A_{2}\right)=\sqrt{\frac{1}{2}\left(\left(\mu_{A_{1}}-\mu_{A_{2}}\right)^{2}+\left(v_{A_{1}}-v_{A_{2}}\right)^{2}\right)} .
$$

With respect to HFSs, Xu and Xia (2011) further defined the hesitant distance measures. For two HFEs $h_{1}$ and $h_{2}$, the hesitant distance measures can be stated as follows:

1) The hesitant normalized Hamming distance

$$
d_{1}\left(h_{1}, h_{2}\right)=\frac{1}{\# h}\left(S_{s}\left(\bigcup_{\gamma_{1}^{\sigma(i)} \in h_{1}, \gamma_{2}^{\sigma(i)} \in h_{2}}\left\{\left|\gamma_{1}^{\sigma(i)}-\gamma_{2}^{\sigma(i)}\right|\right\}\right)\right) ;
$$

2) The hesitant normalized Euclidean distance

$$
d_{2}\left(h_{1}, h_{2}\right)=\left(\frac{1}{\# h}\left(S_{s}\left(\bigcup_{\gamma_{1}^{\sigma(i)} \in h_{1}, \gamma_{2}^{\sigma(i)} \in h_{2}}\left\{\left(\gamma_{1}^{\sigma(i)}-\gamma_{2}^{\sigma(i)}\right)^{2}\right\}\right)\right)\right)^{1 / 2},
$$

where $\# h=\max \left(\# h_{1}, \# h_{2}\right), S_{s}$ is a function that indicates a summation of all values in a set, $\gamma_{1}^{\sigma(i)}$ and $\gamma_{2}^{\sigma(i)}$ are the ith largest values in $h_{1}$ and $h_{2}$ respectively.

To use the hesitant distance measures above, we need to make sure that there is the same number of memberships between two HFEs. Zhu and Xu (2013) developed an optimized parameter $\varsigma(0 \leq \varsigma \leq 1)$ to add linguistic terms in hesitant fuzzy linguistic term sets. Motivated by the optimized parameter, we give the following definition.

Definition 13. For a MU, $u=\left(\gamma_{1}, \ldots, \gamma_{m}\right)$, let $u^{-}=\min \{\gamma \mid \gamma \in u\}$ and $u^{+}=\max \{\gamma \mid \gamma \in u\}$ be the minimum and maximum memberships in $u$ respectively, and $\varsigma(0 \leq \varsigma \leq 1)$ be the optimized parameter, then we call $\psi=\varsigma u^{+}+(1-\varsigma) u^{-}$an added membership.

For two EHFEs with different number of MUs, we further utilize the optimized parameter to obtain a MU.

Definition 14. Given an EHFE, $H_{h_{D}}=\bigcup_{\gamma_{1} \in h_{1}, \ldots, \gamma_{m} \in h_{m}}\left\{\left(\gamma_{1}, \ldots, \gamma_{m}\right)\right\} \quad(D=1, \cdots, m)$, let $h_{D}^{-}$ and $h_{D}^{+}$be the minimum and maximum memberships in $h_{D}$ respectively, and $\varsigma(0 \leq \varsigma \leq 1)$ be the optimized parameter, then an added MU is defined as $t=\left(\boldsymbol{\psi}_{1}, \ldots, \boldsymbol{\psi}_{m}\right)$, where $*_{D}=\varsigma\left(h_{D}^{+}\right)+(1-\varsigma)\left(h_{D}^{-}\right)(D=1, \cdots, m)$.

To compare two MUs, the comparison law can be stated below.

Definition 15. For a MU, $u=\left(\gamma_{1}, \ldots, \gamma_{m}\right)$, then we call $s(u)=(1 / \# u) \sum_{\gamma \in u} \gamma$ the score function of $u$, where $\# u$ is the number of memberships in $u$. For any two MUs, $u_{1}$ and $u_{2}$, if $s\left(u_{1}\right)>s\left(u_{2}\right)$, then $u_{1} \succ u_{2}$; if $s\left(u_{1}\right)=s\left(u_{2}\right)$, then $u_{1} \sim u_{2}$, where " $\succ$ " denotes "be superior to", and " " means "be indifferent to".

Furthermore, we can also consider the deviation degree to compare MUs. A small deviation degree of all elements with respect to the average value in a MU reflects how these elements agree with each other, that is, they have a higher consistency.

Definition 16. For a MU, $u=\left(\gamma_{1}, \ldots, \gamma_{m}\right)$, let $s(u)$ be the score function of $u$, then we call $\rho(u)=\left[(1 / \# u) \sum_{\gamma \in u}(\gamma-s(u))^{2}\right]^{\frac{1}{2}}$ the deviation function of HFSs, where \#u is the number of memberships in $u$.

The deviation function reflects the deviation degree between all possible memberships in a MU and their average value. Based on the score function and the deviation function, we develop the following comparison laws. 
Definition 17. Let $u_{1}$ and $u_{2}$ be two MUs, $s\left(u_{1}\right)$ and $s\left(u_{2}\right)$ the scores of $u_{1}$ and $u_{2}$ respectively, and $\rho\left(u_{1}\right)$ and $\rho\left(u_{2}\right)$ the deviation degrees of $\mathrm{u}_{1}$ and $\mathrm{u}_{2}$ respectively, then:

1) if $s\left(u_{1}\right)<s\left(u_{2}\right)$, then $u_{1} \prec u_{2}$;

2) if $s\left(u_{1}\right)=s\left(u_{2}\right)$, then

(1) if $\rho\left(u_{1}\right)=\rho\left(u_{2}\right)$, then $u_{1} \sim u_{2}$;

(2) if $\rho\left(u_{1}\right)<\rho\left(u_{2}\right)$, then $u_{1} \succ u_{2}$;

(3) if $\rho\left(u_{1}\right)>\rho\left(u_{2}\right)$, then $u_{1} \prec u_{2}$.

For any two EHFEs, $H_{1}=\bigcup_{u_{1} \in H_{1}}\left\{u_{1}\right\}$ and $H_{2}=\bigcup_{u_{2} \in H_{2}}\left\{u_{2}\right\}$, and $\varsigma(0 \leq \varsigma \leq 1)$, we make them have the same number of MUs and the same number of memberships in each MU by Definitions 13 and 14, respectively. According to Definitions 15-17 to rank MUs, and combining the normalized Hamming distance and the normalized Euclidean distance, we define the following distance measures:

1) Extended hesitant Normalized Hamming distance

$d_{h}\left(H_{1}, H_{2}\right)=\frac{1}{(\# H)(\# u)} S_{s}\left(\sum_{i=1}^{\# H} \sum_{j=1}^{\# u}\left(\begin{array}{c}U_{\left(\gamma_{1}\right)^{\sigma(j)} \in u_{1}^{\sigma(i)},\left(\gamma_{2}\right)^{\sigma(j)} \in u_{2}^{\sigma(i)}}\left\{\left|\left(\gamma_{1}\right)^{\sigma(j)}-\left(\gamma_{2}\right)^{\sigma(j)}\right|\right) \\ \left.u_{1}^{\sigma(i)} \in H_{1}, u_{2}^{\sigma(i)} \in H_{2}\right\}\end{array}\right)\right)$;

2) Extended hesitant Normalized Euclidean distance

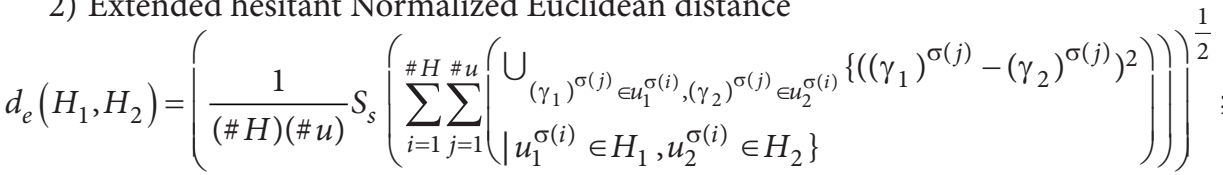

3) Extended hesitant Normalized generalized distance

$d_{g}\left(H_{1}, H_{2}\right)=\left(\frac{1}{(\# H)(\# u)} S_{s}\left(\sum_{i=1}^{\# H} \sum_{j=1}^{\# u}\left(\begin{array}{l}\left.U_{\left(\gamma_{1}\right)^{\sigma(j)} \in u_{1}^{\sigma(i)},\left(\gamma_{2}\right)^{\sigma(j)} \in u_{2}^{\sigma(i)}}\left\{\left(\left(\gamma_{1}\right)^{\sigma(j)}-\left(\gamma_{2}\right)^{\sigma(j)}\right)^{\lambda}\right) H_{1}, u_{2}^{\sigma(i)} \in H_{2}\right\}\end{array}\right)\right)\right)^{\frac{1}{\lambda}} \begin{array}{r}(\lambda>0), \\ (22)\end{array}$

where $\# H=\# H_{1}=\# H_{2}$ ( \# $H_{1}$ and $\# H_{2}$ are the number of MUs in $H_{1}$ and $H_{2}$ respectively), \#u=\# $u_{1}=\# u_{2}, S_{s}$ is a function that indicates a summation of all values in a set, $\left(\gamma_{1}\right)^{\sigma(j)}$ and $\left(\gamma_{2}\right)^{\sigma(j)}$ are the $j$ th largest memberships in $u_{1}$ and $u_{2}$ respectively, $u_{1}^{\sigma(i)}$ and $u_{2}^{\sigma(i)}$ are the ith largest MUs in $H_{1}$ and $H_{2}$ respectively.

It's clear that the extended hesitant generalized normalized distance can reduce to the extended hesitant normalized Hamming distance and the extended hesitant normalized Euclidean distance when $\lambda=1$ and $\lambda=2$ respectively.

Example 2. Let $H_{1}=\{(0.2,0.3),(0.2,0.4),(0.3,0.3),(0.3,0.4)\}$ and $H_{2}=\{(0.3,0.4),(0.1,0.6)\}$ be two EHFEs, and $\varsigma=0.8$. Since $\# H_{1}(=4)>\# H_{2}(=2)$, then we should add two MUs to $\# H_{2}$. According to Definition 14 , we can get the added MU, $t=\left(\not_{1}, \psi_{2}\right)=(0.26,0.56)$, where

$$
\text { 为 }_{1}=0.8 \times 0.3+0.2 \times 0.1=0.26, \text { * }_{2}=0.8 \times 0.6+0.2 \times 0.4=0.56 \text {. }
$$

Thus, an adjusted $\mathrm{H}_{2}$ with the added MUs is:

$$
H_{2}^{\prime}=\bigcup_{u_{2} \in H_{2}}\left\{u_{2}\right\}=\{(0.3,0.4),(0.1,0.6),(0.26,0.56),(0.26,0.56)\} .
$$


By Eqs (20) and (21), we have:

$d_{h}\left(H_{1}, H_{2}\right)=\frac{1}{2 \times 4} S_{s}\left(\sum_{i=1}^{4} \sum_{j=1}^{2}\left(\begin{array}{l}\left.U_{\left(\gamma_{1}\right)^{\sigma(j)} \in u_{1}^{\sigma(i)},\left(\gamma_{2}^{\prime}\right)^{\sigma(j)} \in u_{2}^{\prime \sigma(i)}}\left\{\left|\left(\gamma_{1}\right)^{\sigma(j)}-\left(\gamma_{2}^{\prime}\right)^{\sigma(j)}\right|\right)\right)=0.1250, \\ \left.u_{1}^{\sigma(i)} \in H_{1}, u_{2}^{\prime \sigma(i)} \in H_{2}^{\prime}\right\}\end{array}\right)\right)$
$d_{e}\left(H_{1}, H_{2}\right)=\left(\frac{1}{2 \times 4} S_{s}\left(\sum_{i=1}^{4} \sum_{j=1}^{2}\left(\begin{array}{l}\left.\left.U_{\left(\gamma_{1}\right)^{\sigma(j)} \in u_{1}^{\sigma(i)},\left(\gamma_{2}^{\prime}\right)^{\sigma(j)} \in u_{2}^{\prime \sigma(i)}}\left\{\left(\left(\gamma_{1}\right)^{\sigma(j)}-\left(\gamma_{2}^{\prime}\right)^{\sigma(j)}\right)^{2}\right)\right)\right)^{\frac{1}{2}}=0.1442 . \\ \left.u_{1}^{\sigma(i)} \in H_{1}, u_{2}^{\prime \sigma(i)} \in H_{2}^{\prime}\right\}\end{array}\right)\right)^{\sigma}=\right.$

In Example 2, the distances vary with two parameters, the optimized parameter $\varsigma$, and the parameter $\lambda$. More details can be found in Figure 1 .

From Figure 1, we can partly conclude that the distances between $H_{1}$ and $H_{2}$ increase with the increase of $\varsigma$. However, as $\lambda$ increases, the distances increase approaching the path of the sine function.
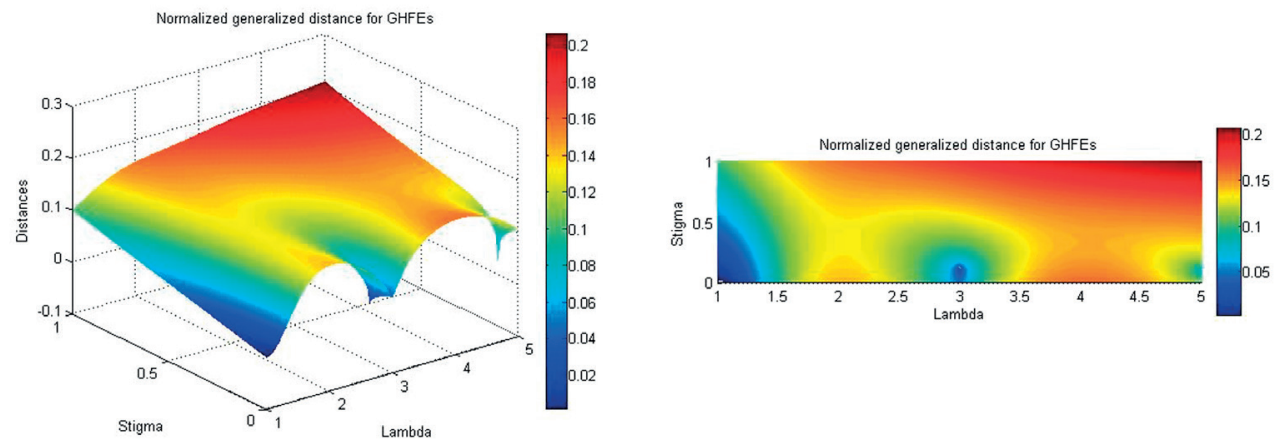

Fig. 1. Extended hesitant Normalized generalized distance $(\lambda \in[1,5], \varsigma \in[0,1])$

\section{Weighted extended hesitant distance measures}

The hesitant distance measures proposed by Xu and Xia (2011) cannot consider the weights of the DMs. To save all the information provided by the DMs, distinct them from each other, and consider their different importance in decision making, we now propose the weighted extended hesitant distance measures in this section.

Assume aroup decision making problem with $m$ DMs. For two EHFEs, $H_{k}=\bigcup_{u_{k} \in H_{k}}\left\{u_{k}\right\}=$ $\bigcup_{\left(\gamma_{1}^{(k)}, \ldots, \gamma_{m}^{(k)}\right) \in H_{k}}\left\{\left(\gamma_{1}^{(k)}, \ldots, \gamma_{m}^{(k)}\right)\right\} \quad(k=1,2)$, the weights of DMs are $\omega_{D} \quad(D=1, \ldots, m)$ with $\omega_{D} \in[0,1]$ and $\sum_{D=1}^{m} \omega_{D}=1$. Let $\gamma_{\omega_{D}}^{(k)}=\omega_{D} \gamma_{D}^{(k)}(D=1, \ldots, m)$ be memberships associated with the DMs' weights. According to the extended hesitant generalized distance, we now develop a weighted extended hesitant generalized distance as:

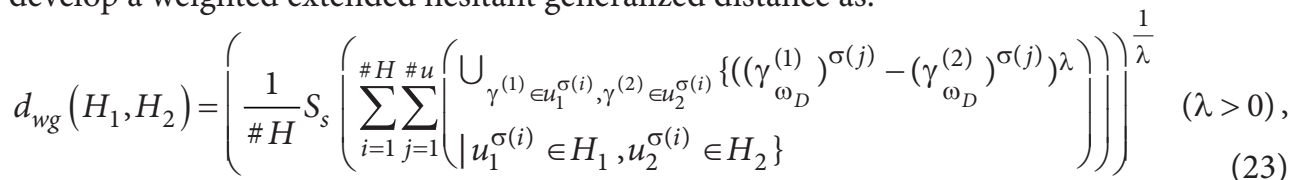


where $\left(\gamma_{\omega_{D}}^{(1)}\right)^{\sigma(j)}$ and $\left(\gamma_{\omega_{D}}^{(2)}\right)^{\sigma(j)}$ are the jth largest memberships associated with the DMs' weights in $u_{1}$ and $u_{2}$ respectively.

In particular, if $\lambda=1$ and $\lambda=2$, then the weighted extended hesitant generalized distance reduces to a weighted extended hesitant weighted Hamming distance and a weighted extended hesitant Euclidean distance respectively.

To emphasize the importance of DMs in group decision making further, we now combine the weighted extended hesitant distance measures with the Dempster-Shafer belief structure (Dempster 1967; Shafer 1976). The Dempster-Shafer belief structure has proven to be a useful tool for representing uncertainty, which has been used in an astonishingly wide range of applications (Yager 1992; Yager et al. 1994).

Definition 18 (Dempster 1967; Shafer 1976). A Dempster-Shafer belief structure consists of a collection of $r$ non-null subsets $B_{k} \quad(k=1,2, \cdots, r)$ of $X$ defined on a space $X=\left\{x_{1}, x_{2}, \ldots, x_{n}\right\}$, called focal elements, and a mapping $p$ called the probability assignment, defined as $p: 2^{X} \rightarrow[0,1]$ such that:

1) $p\left(B_{j}\right) \in[0,1]$;

2) $\sum_{k=1}^{r} p\left(B_{k}\right)=1$;

3) $p(C)=0, \forall C \neq B_{k}$.

Assume a group decision making problem associated with $m$ DMs with a collection of weights $w_{D}(D=1, \ldots, m)$, and a set of the alternatives, $\left\{A_{1}, \ldots, A_{q}\right\}$, with the set of states of nature $\left\{S_{1}, \ldots, S_{n}\right\}$. The DMs provide all possible preferences over all the alternatives $A_{i}$ $(i=1, \ldots, q)$ with respect to the states of nature (criteria) $S_{j}(j=1,2, \ldots, n)$, then we can get $q \times n$ EHFEs $H_{i j}(i=1, \ldots, q ; j=1, \ldots, n)$, which indicates the group preferences over the alternative $A_{i}$ of the criterion $S_{j}$.

Let $C_{i j}$ be a payoff to the alternative $A_{i}$ and the state of nature is $S_{j}, C=\left(C_{i j}\right)_{q \times n}$ a payoff matrix, and $\varsigma(0 \leq \varsigma \leq 1)$ the optimized parameter. The DMs' knowledge of the states of nature is captured in terms of a belief structure $p$ with the focal elements $B_{1}, B_{2}, \ldots, B_{r}$, each of which is associated with a weight $p\left(B_{k}\right)$, where $\sum_{k=1}^{r} p\left(B_{k}\right)=1$. We now develop the following approach to deal with group decision making.

Step 1. Construct the extended hesitant fuzzy decision matrix $H=\left(H_{i k}\right)_{q \times n}$ by EHFEs, $H_{i j}(i=1, \ldots, q ; j=1, \ldots, n)$.

Step 2. Assume a standard EHFE $\mathrm{H}^{*}$, and the optimized parameter, then calculate the distance between $H^{\star}$ and $H_{i j}$ by the extended hesitant distance measures or the weighted extended hesitant distance measure. Let $C_{i j}$ equal to the distance, and construct the payoff matrix $C=\left(C_{i j}\right)_{q \times n}$.

Step 3. Calculate the belief function $p$ about the states of nature.

Step 4. Utilize the optimized parameter to calculate the collection of weights (O'Hagan 1988; Yager 1993) which are used in the OWA aggregation for each cardinality of focal elements.

Step 5. Determine the payoff collection, $M_{i k}=\left\{C_{i j} \mid S_{j} \in B_{k}\right\}$, which is a set of payoffs that are possible if we select the alternative $A_{\mathrm{i}}$ and the focal element $B_{k}$ occurs, and calculate the aggregated payoff, $V_{i k}=O W A\left(M_{i k}\right)$. 
Step 6. Calculate $C_{i}=\sum_{k=1}^{r} V_{i k} p\left(B_{k}\right)$, and select the alternative which has the best generalized expected value as the optimal alternative.

Example 3 (Kahraman, Kaya 2010). Energy is an indispensable factor for the social-economic development of societies. Thus the correct energy policy affects economic development and environment, the most appropriate energy policy selection is very important. Suppose that there are five alternatives (energy projects) $A_{i}(i=1,2,3,4,5)$ to be invested, and four criteria to be considered: $S_{1}$ - technological; $S_{2}$ - environmental; $S_{3}$ - socio-political; $S_{4}$ - economic. Five DMs are invited to evaluate the performances of the five alternatives.

$\mathrm{Xu}$ and Xia (2011) used HFSs to collect the DMs' preferences and utilized the hesitant distance measures to deal with this problem. The precondition of the method introduced by $\mathrm{Xu}$ and Xia (2011) is that the DMs should give their preferences anonymously so as to ignore the repeated preferences. To deal with this energy policy problem without information loss, consider weights of the DMs in group decision making, and compare Xu and Xia (2011)'s resolution, we now give the following approach to deal with this problem.

Step 1. The DMs $D_{k}(k=1,2,3,4,5)$ provide their preferences over all the alternatives $A_{\mathrm{i}}$ $A_{i}(i=1,2, \ldots, 5)$ with respect to the criteria $S_{j}(j=1,2,3,4)$ based on HFSs, then we can construct EHFSs, and get an extended hesitant fuzzy matrix $H=\left(H_{i j}\right)_{5 \times 4}$, which indicates the group preferences over the alternative $A_{i}$ of the criterion $S_{\mathrm{j}}$. Assume that the matrix is shown in Table 1.

Table 1. Extended hesitant fuzzy decision matrix

\begin{tabular}{ccc}
\hline & $S_{1}$ & $S_{2}$ \\
\hline$A_{1}$ & $\{(0.3,0.4,0.3,0.4,0.5)\}$ & $\{(0.7,0.8,0.3,0.8,0.6),(0.7,0.8,0.4,0.8,0.6)\}$ \\
\hline$A_{2}$ & $\{(0.3,0.4,0.5,0.2,0.5),(0.3,0.4,0.5,0.3,0.5)\}$ & $\{(0.5,0.6,0.5,0.6,0.6)\}$ \\
\hline$A_{3}$ & $\{(0.4,0.5,0.5,0.5,0.6)\}$ & $\left\{\begin{array}{l}(0.5,0.6,0.7,0.6,0.5),(0.6,0.6,0.7,0.6,0.5), \\
(0.5,0.6,0.8,0.6,0.5),(0.6,0.6,0.8,0.6,0.5)\end{array}\right\}$ \\
\hline$A_{4}$ & $\{(0.3,0.2,0.2,0.3,0.1)\}$ & $\{(0.6,0.5,0.7,0.5,0.5)\}$ \\
\hline$A_{5}$ & $\{(0.3,0.4,0.6,0.2,0.2),(0.3,0.3,0.6,0.2,0.2)\}$ & $\{(0.6,0.8,0.5,0.4,0.6),(0.6,0.8,0.5,0.5,0.6)\}$ \\
\hline & \multicolumn{1}{c}{$S_{3}$} & $S_{4}$ \\
\hline$A_{1}$ & $\left\{\begin{array}{l}(0.3,0.4,0.2,0.3,0.2),(0.4,0.4,0.2,0.3,0.2), \\
(0.3,0.4,0.3,0.3,0.2),(0.4,0.4,0.3,0.3,0.2)\end{array}\right.$ & $\{(0.6,0.5,0.5,0.4,0.6)\}$ \\
\hline$A_{2}$ & $\{(0.6,0.4,0.5,0.3,0.5),(0.6,0.4,0.4,0.3,0.5)\}$ & $\{(0.3,0.4,0.5,0.2,0.2),(0.3,0.4,0.4,0.2,0.2)\}$ \\
\hline$A_{3}$ & $\{(0.7,0.3,0.9,0.8,0.6),(0.7,0.3,0.8,0.8,0.6)\}$ & $\{(0.7,0.8,0.7,0.8,0.8)\}$ \\
\hline$A_{4}$ & $\{(0.4,0.3,0.2,0.3,0.5)\}$ & $\{(0.3,0.2,0.7,0.2,0.1)\}$ \\
\hline$A_{5}$ & $\{(0.7,0.5,0.6,0.8,0.6)\}$ & $\{(0.6,0.4,0.5,0.4,0.6),(0.7,0.4,0.5,0.4,0.6)\}$ \\
\hline
\end{tabular}


Step 2. Let $A^{*}=\{(1,1,1,1,1)\}$ be the ideal values of alternative seen as a standard EHFE $H^{*}$, $\varsigma=0.75$ be the optimized parameter, $w=(0.3,0.1,0.3,0.2,0.1)$ be the weighting vector of the DMs, and $\lambda=1$. By Eq. (23), we can calculate the distance between $H^{*}$ and $H_{i j}$, i.e. $d_{w g}\left(H^{*}, H_{i j}\right)$. Let $C_{i j}=d_{w g}\left(H^{*}, H_{i j}\right)$, then construct the payoff matrix $C=\left(C_{i j}\right)_{5 \times 4}$, shown in Table 2.

Table 2. The payoff matrix

\begin{tabular}{ccccc}
\hline & $\mathrm{S}_{1}$ & $\mathrm{~S}_{2}$ & $\mathrm{~S}_{3}$ & $\mathrm{~S}_{4}$ \\
\hline$A_{1}$ & 0.6800 & 0.4000 & 0.7750 & 0.5000 \\
\hline$A_{2}$ & 0.6550 & 0.4900 & 0.5700 & 0.7600 \\
\hline$A_{3}$ & 0.5500 & 0.4550 & 0.4000 & 0.2700 \\
\hline$A_{4}$ & 0.8700 & 0.4800 & 0.7000 & 0.7800 \\
\hline$A_{5}$ & 0.7400 & 0.4350 & 0.4100 & 0.5050 \\
\hline
\end{tabular}

Step 3. The DMs analyze the energy policy problem so as to obtain the probabilistic information about the states of nature. Assume that the DMs' knowledge of the states of nature consists of the following belief structure, shown in Table 3.

Table 3. Belief structure

\begin{tabular}{cc}
\hline Focal element & Weights \\
\hline$B_{1}=\left\{S_{1}, S_{3}\right\}$ & 0.15 \\
\hline$B_{2}=\left\{S_{2}, S_{4}\right\}$ & 0.25 \\
\hline$B_{3}=\left\{S_{1}, S_{3}, S_{4}\right\}$ & 0.6 \\
\hline
\end{tabular}

Step 4. We use the O'Hagan (1988) method to obtain weighting vectors associated with the OWA operators for various numbers of arguments. Since $\varsigma=0.75$, then we can get the weighting vectors shown in Table 4.

Table 4. Weighting vectors for various numbers of arguments

\begin{tabular}{cccc}
\hline Number of arguments & $\mathrm{w}_{1}$ & $\mathrm{w}_{2}$ & $\mathrm{w}_{3}$ \\
\hline 2 & 0.75 & 0.25 & \\
\hline 3 & 0.62 & 0.27 & 0.11 \\
\hline
\end{tabular}

Step 5. Since $M_{i k}=\left\{C_{i j} \mid S_{j} \in B_{k}\right\}$ and $V_{i k}=O W A\left(M_{i k}\right)$, then we can get $V_{i k}$ for all $i$ and $k(i=1,2,3,4,5 ; k=1,2,3)$.

Step 6. Since $C_{i}=\sum_{k=1}^{3} V_{i k} p\left(B_{k}\right)$, and according to $V_{i k}$ and the belief structure, we have $C_{1}=0.5884, C_{2}=0.5801, C_{3}=0.4135, C_{4}=0.6617, C_{5}=0.5390$. Thus, $A_{3}$ is the optimal alternative closest to the ideal values of alternative with the minimum generalized expected value, $C_{3}=0.4135$. 
According to the approach above, we know that $C_{i}(i=1,2,3,4,5)$ vary with the parameter $\lambda$. For different $\lambda$, we get Table 5 below.

Table 5. Generalized expected values for different $\lambda$

\begin{tabular}{ccccccc}
\hline & $\mathrm{C}_{1}$ & $\mathrm{C}_{2}$ & $\mathrm{C}_{3}$ & $\mathrm{C}_{4}$ & $\mathrm{C}_{5}$ & Rankings \\
\hline$\lambda=1$ & 0.5884 & 0.5801 & 0.4135 & 0.6617 & 0.5390 & $A_{4} \succ A_{1} \succ A_{2} \succ A_{5} \succ A_{3}$ \\
\hline$\lambda=2$ & 0.3006 & 0.2873 & 0.2069 & 0.3221 & 0.2663 & $A_{4} \succ A_{1} \succ A_{2} \succ A_{5} \succ A_{3}$ \\
\hline$\lambda=5$ & 0.2258 & 0.2074 & 0.1531 & 0.2315 & 0.1922 & $A_{4} \succ A_{1} \succ A_{2} \succ A_{5} \succ A_{3}$ \\
\hline
\end{tabular}

It's clear that for the three different values of $\lambda$, the same ranking result can be obtained in Example 3. In practice, we let $\lambda=1$ without loss of generality.

The DMs influence the final decision result due to their different importance, if we let $w^{\prime}=(0.2,0.2,0.2,0.2,0.2)$ be the weighting vector of the DMs, which means that there is no difference among the DMs, we can obtain a different ranking result. By the developed approach, and all other conditions are still the same, we have $C_{1}=0.5673, C_{2}=0.5764$, $C_{3}=0.4179, C_{4}=0.6820, C_{5}=0.5463$, and the ranking $A_{4} \succ A_{2} \succ A_{1} \succ A_{5} \succ A_{3}$, where a change in ranking happens between $A_{1}$ and $A_{2}$.

The hesitant distance measures cannot consider the differences among the DMs, which is similar to the situation that the weighting vector of DMs is $w^{\prime}=(0.2,0.2,0.2,0.2,0.2)$. For comparison, we apply the hesitant distance measures to Example 3. According to the definition of reduced EHFEs, we first transform Table 1 to a hesitant fuzzy decision matrix, shown in Table 6.

Table 6. Hesitant fuzzy decision matrix

\begin{tabular}{ccccc}
\hline & $\mathrm{S}_{1}$ & $\mathrm{~S}_{2}$ & $\mathrm{~S}_{3}$ & $\mathrm{~S}_{4}$ \\
\hline$A_{1}$ & $\{0.3,0.4,0.5\}$ & $\{0.7,0.8,0.3,0.6,0.4\}$ & $\{0.3,0.4,0.2\}$ & $\{0.6,0.5,0.4\}$ \\
\hline$A_{2}$ & $\{0.3,0.4,0.5,0.2\}$ & $\{0.5,0.6\}$ & $\{0.6,0.4,0.5,0.3\}$ & $\{0.3,0.4,0.5,0.2\}$ \\
\hline$A_{3}$ & $\{0.4,0.5,0.6\}$ & $\{0.5,0.6,0.7,0.8\}$ & $\{0.7,0.3,0.9,0.8,0.6\}$ & $\{0.7,0.8\}$ \\
\hline$A_{4}$ & $\{0.3,0.2,0.1\}$ & $\{0.6,0.5,0.7\}$ & $\{0.4,0.3,0.2,0.5\}$ & $\{0.3,0.2,0.7,0.1\}$ \\
\hline$A_{5}$ & $\{0.3,0.4,0.6,0.2\}$ & $\{0.6,0.8,0.5,0.4\}$ & $\{0.7,0.5,0.6,0.8\}$ & $\{0.6,0.4,0.5,0.7\}$ \\
\hline
\end{tabular}

With other conditions still being the same, and according to the hesitant normalized Hamming distance, we have $C_{1}=0.7043, C_{2}=0.7153, C_{3}=0.5869, C_{4}=0.7851, C_{5}=0.6201$, and the ranking $A_{4} \succ A_{2} \succ A_{1} \succ A_{5} \succ A_{3}$, which is the same as the ranking result when the weighting vector of the DMs is $w^{\prime}=(0.2,0.2,0.2,0.2,0.2)$. Thus, the existing hesitant distance measures can be considered as a particular case of the extended hesitant distance measures with certain conditions. And the extended hesitant distance measures appear to be more extensive and effective in practical applications. 


\section{Conclusions}

We have developed the extended hesitant fuzzy sets (EHFSs) to resolve the information loss problem of hesitant fuzzy sets (HFSs) in this paper, and have shown that intuitionistic fuzzy sets (IFSs), HFSs and dual hesitant fuzzy sets (DHFSs) are particular cases of EHFSs with certain conditions. EHFSs can also be represented as fuzzy multisets (FMSs) or type-2 fuzzy sets (T2FSs). Given several hesitant fuzzy elements (HFEs), we can construct an extended hesitant fuzzy element (EHFE) by their Cartesian product. On the contrary, given an EHFE, we can get its reduced EHFE which is a HFE. As an extension of HFSs, EHFSs increase the richness of numerical representation based on the membership units (MUs), enhance the modeling abilities of HFSs, and can identify different DMs in group decision making. We have further developed some extended hesitant distance measures which take advantages of EHFSs without the information loss problem. A weighted extended hesitant distance measure has been developed, which can take the different importance of the DMs into account in group decision making comparing with the existing hesitant distance measures. Combining the proposed weighted extended hesitant distance measure with Dempster-Shafer belief structure, we have proposed an approach to deal with group decision making problems with an illustrative example. In the future, EHFSs are likely to play an importance role in group decision making with more studies on the theory and applications.

\section{Acknowledgements}

The authors would like to thank the anonymous referees for their insightful and constructive comments and suggestions that have led to an improved version of this paper. The work was supported by the Project Funded by China Postdoctoral Science Foundation.

\section{References}

Atanassov, K. 1986. Intuitionistic fuzzy sets, Fuzzy sets and Systems 20(1): 87-96. http://dx.doi.org/10.1016/S0165-0114(86)80034-3

Atanassov, K.; Gargov, G. 1989. Interval valued intuitionistic fuzzy sets, Fuzzy sets and systems 31(3): 343-349. http://dx.doi.org/10.1016/0165-0114(89)90205-4

Baležentis, A.; Baležentis, T.; Misiunas, A. 2012. An integrated assessment of Lithuanian economic sectors based on financial ratios and fuzzy MCDM methods, Technological and Economic Development of Economy 18(1): 34-53. http://dx.doi.org/10.3846/20294913.2012.656151

Bustince, H.; Burillo, P. 1995. Correlation of interval-valued intuitionistic fuzzy sets, Fuzzy Sets and Systems 74(2): 237-244. http://dx.doi.org/10.1016/0165-0114(94)00343-6

Chaudhuri, B. B.; Rosenfeld, A. 1999. A modified Hausdorff distance between fuzzy sets, Information Sciences 118(1): 159-171. http://dx.doi.org/10.1016/S0020-0255(99)00037-7

Chen, T.-Y.; Wang, H.-P.; Lu, Y.-Y. 2011. A multicriteria group decision-making approach based on interval-valued intuitionistic fuzzy sets: a comparative perspective, Expert Systems with Applications 38(6): 7647-7658. http://dx.doi.org/10.1016/j.eswa.2010.12.096

Dempster, A. P. 1967. Upper and lower probabilities induced by a multivalued mapping, The Annals of Mathematical Statistics 38(2): 325-339. http://dx.doi.org/10.1214/aoms/1177698950 
Diamond, P.; Kloeden, P. E. 1994. Metric spaces of fuzzy sets: theory and applications. World Scientific Publishing Company. http://dx.doi.org/10.1142/2326

Doctor, F.; Hagras, H.; Callaghan, V. 2005. A type-2 fuzzy embedded agent to realise ambient intelligence in ubiquitous computing environments, Information Sciences 171(4): 309-334. http://dx.doi.org/10.1016/j.ins.2004.09.008

Dubois, D. J. 1980. Fuzzy sets and systems: theory and applications. Academic press.

Greenfield, S.; Chiclana, F.; Coupland, S.; John, R. 2009. The collapsing method of defuzzification for discretised interval type-2 fuzzy sets, Information Sciences 179(13): 2055-2069. http://dx.doi.org/10.1016/j.ins.2008.07.011

Hagras, H. A. 2004. A hierarchical type-2 fuzzy logic control architecture for autonomous mobile robots, IEEE Transactions on Fuzzy Systems 12(4): 524-539. http://dx.doi.org/10.1109/TFUZZ.2004.832538

Kacprzyk, J. 1997. Multistage fuzzy control: a prescriptive approach. John Wiley \& Sons, Inc.

Kahraman, C.; Kaya, İ. 2010. A fuzzy multicriteria methodology for selection among energy alternatives, Expert Systems with Applications 37(9): 6270-6281. http://dx.doi.org/10.1016/j.eswa.2010.02.095

Karnik, N. N.; Mendel, J. M. 2001. Centroid of a type-2 fuzzy set, Information Sciences 132(1): 195-220. http://dx.doi.org/10.1016/S0020-0255(01)00069-X

Li, D.; Cheng, C. 2002. New similarity measures of intuitionistic fuzzy sets and application to pattern recognitions, Pattern Recognition Letters 23(1): 221-225.

Liu, H.-W.; Wang, G.-J. 2007. Multi-criteria decision-making methods based on intuitionistic fuzzy sets, European Journal of Operational Research 179(1): 220-233.

http://dx.doi.org/10.1016/j.ejor.2006.04.009

Merigó, J. M.; Casanovas, M. 2009. Induced aggregation operators in decision making with the DempsterShafer belief structure, International Journal of Intelligent Systems 24(8): 934-954. http://dx.doi.org/10.1002/int.20368

Merigó, J. M.; Gil-Lafuente, A. M. 2009. The induced generalized OWA operator, Information Sciences 179(6): 729-741. http://dx.doi.org/10.1016/j.ins.2008.11.013

Miyamoto, S. 2003. Information clustering based on fuzzy multisets, Information Processing \& Management 39(2): 195-213. http://dx.doi.org/10.1016/S0306-4573(02)00047-X

Mizumoto, M.; Tanaka, K. 1976. Some properties of fuzzy sets of type 2, Information and Control 31(4): 312-340. http://dx.doi.org/10.1016/S0019-9958(76)80011-3

O'Hagan, M. 1988. Aggregating template or rule antecedents in real-time expert systems with fuzzy set logic, in Twenty-Second Asilomar Conference on Signals, Systems and Computers, 31 October - 2 November 1998, Pacific Grove, California, IEEE, 681-689. http://dx.doi.org/10.1109/ACSSC.1988.754637

Rickard, J. T.; Aisbett, J.; Gibbon, G. 2009. Fuzzy subsethood for fuzzy sets of type-2 and generalized type-n, IEEE Transactions on Fuzzy Systems 17(1): 50-60. http://dx.doi.org/10.1109/TFUZZ.2008.2006369

Shafer, G. 1976. A mathematical theory of evidence. Princeton: Princeton university press.

Stankevičienè, J.; Mencaite, E. 2012. The evaluation of bank performance using a multicriteria decision making model: a case study on Lithuanian commercial banks, Technological and Economic Development of Economy 18(1): 189-205. http://dx.doi.org/10.3846/20294913.2012.668373

Szmidt, E.; Kacprzyk, J. 2000. Distances between intuitionistic fuzzy sets, Fuzzy Sets and Systems 114(3): 505-518. http://dx.doi.org/10.1016/S0165-0114(98)00244-9

Torra, V. 2010. Hesitant fuzzy sets, International Journal of Intelligent Systems 25: 529-539.

Wang, T.; Lu, Z.; Li, F. 2004. Bidirectional approximate reasoning based on weighted similarity measures of vague set, Minimicro Systems 25(2): 211-215. Shenyang.

Wang, W.; Xin, X. 2005. Distance measure between intuitionistic fuzzy sets, Pattern Recognition Letters 26(13): 2063-2069. http://dx.doi.org/10.1016/j.patrec.2005.03.018 
Wang, Z.; Li, K. W.; Wang, W. 2009. An approach to multiattribute decision making with interval-valued intuitionistic fuzzy assessments and incomplete weights, Information Sciences 179(17): 3026-3040. http://dx.doi.org/10.1016/j.ins.2009.05.001

Wei, G.; Zhao, X.; Wang, H. 2012. An approach to multiple attribute group decision making with interval intuitionistic trapezoidal fuzzy information, Technological and Economic Development of Economy 18(2): 317-330. http://dx.doi.org/10.3846/20294913.2012.676995

Wu, W.; Kou, G.; Peng, Y.; Ergu, D. 2012. Improved AHP-group decision making for investment strategy selection, Technological and Economic Development of Economy 18(2): 299-316. http://dx.doi.org/10.3846/20294913.2012.680520

Xia, M.; Xu, Z. 2011. Hesitant fuzzy information aggregation in decision making, International Journal of Approximate Reasoning 52(3): 395-407. http://dx.doi.org/10.1016/j.ijar.2010.09.002

Xu, Z. 2007a. Intuitionistic fuzzy aggregation operators, IEEE Transactions on Fuzzy Systems 15(6): 1179-1187. http://dx.doi.org/10.1109/TFUZZ.2006.890678

$\mathrm{Xu}, \mathrm{Z}$. 2007b. Some similarity measures of intuitionistic fuzzy sets and their applications to multiple attribute decision making, Fuzzy Optimization and Decision Making 6(2): 109-121. http://dx.doi.org/10.1007/s10700-007-9004-z

$\mathrm{Xu}, \mathrm{Z} .2010$. A method based on distance measure for interval-valued intuitionistic fuzzy group decision making, Information Sciences 180(1): 181-190. http://dx.doi.org/10.1016/j.ins.2009.09.005

Xu, Z.; Chen, J.; Wu, J. 2008. Clustering algorithm for intuitionistic fuzzy sets, Information Sciences 178(19): 3775-3790. http://dx.doi.org/10.1016/j.ins.2008.06.008

Xu, Z.; Xia, M. 2011. Distance and similarity measures for hesitant fuzzy sets, Information Sciences 181(11): 2128-2138. http://dx.doi.org/10.1016/j.ins.2011.01.028

Xu, Z.; Yager, R. R. 2006. Some geometric aggregation operators based on intuitionistic fuzzy sets, International Journal of General Systems 35(4): 417-433. http://dx.doi.org/10.1080/03081070600574353

Yager, R. R. 1986. On the theory of bags, International Journal of General System 13(1): 23-37 http://dx.doi.org/10.1080/03081078608934952.

Yager, R. R. 1988. On ordered weighted averaging aggregation operators in multicriteria decisionmaking, IEEE Transactions on Systems, Man and Cybernetics 18(1): 183-190. http://dx.doi.org/10.1109/21.87068

Yager, R. R. 1992. Decision making under Dempster-Shafer uncertainties, International Journal of General System 20(3): 233-245. http://dx.doi.org/10.1080/03081079208945033

Yager, R. R. 1993. Families of OWA operators, Fuzzy Sets and Systems 59(2): 125-148. http://dx.doi.org/10.1016/0165-0114(93)90194-M

Yager, R. R.; Kacprzyk, J.; Fedrizzi, M. 1994. Advances in the Dempster-Shafer theory of evidence. John Wiley \& Sons, Inc.

Zadeh, L. A. 1965. Fuzzy sets, Information and Control 8: 338-353. http://dx.doi.org/10.1016/S0019-9958(65)90241-X

Zadeh, L. A. 1975. The concept of a linguistic variable and its application to approximate reasoning - I, Information Sciences 8(3): 199-249. http://dx.doi.org/10.1016/0020-0255(75)90036-5

Zhu, B.; Xu, Z. 2013. Consistency measures for hesitant fuzzy linguistic preference relations, IEEE Transactions on Fuzzy Systems 22(1): 35-45. http://dx.doi.org/10.1109/TFUZZ.2013.2245136

Zhu, B.; Xu, Z.; Xia, M. 2012a. Hesitant fuzzy geometric Bonferroni means, Information Sciences 205: 72-85. http://dx.doi.org/10.1016/j.ins.2012.01.048

Zhu, B.; Xu, Z. S.; Xia, M. M. 2012b. Dual hesitant fuzzy sets, Journal of Applied Mathematics. Article ID $879629,13 \mathrm{p}$. 
Bin ZHU received the Bachelor and the PhD degrees in School of Economics and Management, Southeast University, Nanjing, China. He is currently a Postdoctoral Researcher with the School of Economics and Management, Tsinghua University, Beijing, China. His research interests include decision analysis, fuzzy sets and behavior decision making. His research results have been published in European Journal of Operational Research, Information Sciences, IEEE Transactions on Fuzzy Systems, IEEE Transactions on Cybernetics, Fuzzy Sets and Systems, among others.

Zeshui XU (M'08-SM'09) received the PhD degree in management science and engineering from Southeast University, Nanjing, China, in 2003. From April 2003 to May 2005, he was a Postdoctoral Researcher with the School of Economics and Management, Southeast University. From October 2005 to December 2007, he was a Postdoctoral Researcher with the School of Economics and Management, Tsinghua University, Beijing, China. He is currently a Chair Professor with the College of Sciences, PLA University of Science and Technology, Nanjing. He is a member of the Editorial Boards of Information: An International Journal, the International Journal of Applied Management Science, the International Journal of Data Analysis Techniques and Strategies, the Journal of Applied and Computational Mathematics, the International Journal of Research in Industrial Engineering, the System Engineering - Theory and Practice, the Fuzzy Systems and Mathematics, the Journal of Systems Engineering, and the Chinese Journal of Management Science. He has authored the following books: Uncertain Multiple Attribute Decision Making: Methods and Applications (Tsinghua Univ. Press, 2004), Intuitionistic Fuzzy Information Aggregation: Theory and Applications (Science Press and Springer, 2012), and Linguistic Decision Making: Theory and Methods (Science Press and Springer, 2012). He has contributed more than 300 journal articles to professional journals. His current research interests include information fusion, group decision making, computing with words, and aggregation operators. 